\title{
Simulated Brain Tumor Growth Dynamics Using a Three-Dimensional Cellular Automaton
}

\author{
A.R. Kansal ${ }^{1}$ and S. Torquato ${ }^{1,2}$ \\ ${ }^{1}$ Department of Chemical Engineering, ${ }^{2}$ Department of Chemistry and Princeton Materials \\ Institute, Princeton University, Princeton, NJ 08544
}

\begin{abstract}
The competition between local and global driving forces is significant in a wide variety of naturally occurring branched networks. We have investigated the impact of a global minimization criterion versus a local one on the structure of spanning trees. To do so, we consider two spanning tree structures - the generalized minimal spanning tree (GMST) defined by Dror et al. [1] and an analogous structure based on the invasion percolation network, which we term the generalized invasive spanning tree or GIST. In general, these two structures represent extremes of global and local optimality, respectively. Structural characteristics are compared between the GMST and GIST for a fixed lattice. In addition, we demonstrate a method for creating a series of structures which enable one to span the range between these two extremes. Two structural characterizations, the occupied edge density (i.e., the fraction of edges in the graph that are included in the tree) and the tortuosity of the arcs in the trees, are shown to correlate well with the degree to which an intermediate structure resembles the GMST or GIST. Both characterizations are straightforward to determine from an image and are potentially useful tools in the analysis of the formation of network structures.
\end{abstract}

\section{Introduction}

The purpose of the present research is to detail a new method by which information extracted from a single, fixed network structure can be utilized to understand the physical processes which guided the formation of that structure. There are a variety of structures in nature and biology whose temporal development is difficult to observe. Accordingly, the principle data from which to understand the physics that drove the formation of these structures is the final structure itself.

An excellent example of the use of a final network structure to study the underlying physics is the work of Rodriguez-Iturbe and Rinaldo on river basins [2]. Detailed investigations of the structure of river basins combined with a variety of simulation and theoretical analysis support the conclusion that minimal energy dissipation is the driving force (both 


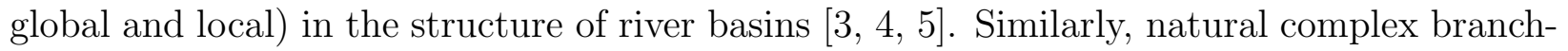
ing patterns are observed in systems as diverse as retinal neurons [6], dielectric breakdown 17] and human vasculature [8]. Another recent example is the network of invading cells in malignant brain tumors observed in vitro [9].

All of these problems can be mapped to the language of spanning trees. For example, in the case of invading tumor cells, the tumor cells form branched chains, i.e., tree structures. The brain offers these invading cells a variety of pathways they can invade along (such as blood vessel and white fiber tracts) which may be interpreted as the edges of an underlying graph, with the various resistances along these pathways playing the role of edge weights. In many of these cases, the underlying physics behind the formation of the observed patterns are only beginning to be understood. The work presented here offers a useful tool in studying the driving forces in the formation of these structures.

Here we consider the class of structures called spanning trees. Formally, spanning trees are defined on graphs and, in the most basic definition, are a loopless, connected set of edges that connect all of the nodes in the underlying graph (see Figure [1). Many different spanning trees can be generated for any given graph. Therefore, it is possible to introduce minimization criteria on the spanning-tree problem and select only those trees which satisfy the criteria. Thus spanning trees represent an excellent test case for investigating the relation between individual structures and the minimization criteria that govern their formation.

A broadly useful class of spanning trees (for examples see refs. [10, 11, 12]), is the minimal weight spanning tree (MST) [13, 14]. The MST is defined on an underlying graph whose edges each have some weight assigned to them. The MST is then the spanning tree (a subset of the edges in the underlying graph) that minimizes the total weight of the edges it includes. The minimal weight spanning tree represents a structure whose formation is guided by a global optimization principle. It is also possible to define other types of criteria for spanning trees. For example, it is possible to define a spanning tree such that only the lowest weight edges at each node are used (a detailed discussion of such a class of structures follows), giving a system with purely local criteria. Other types of criteria can also be imposed, such as the degree constrained minimum spanning tree [15, 16], but are not considered here.

One of the structures we study in this paper is the generalized minimal spanning tree (GMST), proposed by Dror et al. [1]. The GMST is useful in considering problems in which there are relevant length scales longer than a single edge. For example, a biological system is characterized by the diameter of a cell (mapped to a graph edge) as well as the length scale of diffusion in the system, which might be several cell diameters. As the name suggests, the GMST is a generalization of the MST. The GMST is defined on a graph in which the nodes have been partitioned into groups. The spanning condition for the GMST is redefined (relative to the MST) such that instead of requiring that every node in the graph be included in the tree, the inclusion of at least one node from each group is required. The 
GMST structure is the tree that meets this definition of spanning and minimizes the total weight of the edges it includes. When each group contains only one node, the GMST reduces to the MST.

The second class of trees considered in the present work is our generalization of the invasion percolation network [17] that we call the generalized invasive spanning tree (GIST). The invasion percolation network begins with a connected cluster of edges (in the simplest case, this could be just one edge). This cluster then "invades" the remaining edges by taking one edge from the boundary of the cluster and including it in the cluster. The edge that is included is the single edge, of those on the cluster boundary, with the lowest weight. Additional edges are then included, one at a time, in the same fashion until the cluster percolates (spans) across the system. The generalization of the invasion percolation network to the GIST, in analogy to that of the GMST, partitions the nodes into groups and modifies the percolation condition, such that one node from each group must be spanned.

For graphs in which each group is a single node (i.e. those graphs for which the GMST reduces to the MST), the GIST reduces to an acyclic invasion percolation network (i.e., an invasion percolation network without loops) [18]. It has been shown that the acyclic invasion percolation network is identical to the MST [19, 18]. Thus for graphs in which each group is a single node, the GMST and GIST are equivalent structures. Because of this equivalence, it is necessary to consider the GMST and GIST with groups of more than one node, rather than only the MST and invasion percolation, to understand the relation between local and global minimization criteria on tree structures.

The GMST and GIST structures were chosen because they generally offer extremes of global and local criteria. Both classes of trees have criteria which dictate the weight of edges chosen. The GMST structures choose the edges that minimize the total weight of the structure, even if that forces a higher weight edge to be chosen locally. In contrast, the GIST structures include the lowest weight edge locally, even if this results in a higher total weight for the entire tree. Except in the case of single-node groups noted above, each criterion results in a different final structure (though by definition both yield spanning trees). By comparing these structures, the effect of each type of criteria can be identified. Moreover, we provide a method to change a GIST structure incrementally into a more globally optimal GMST-like structure. This allows various structural features to be observed as a function of the degree to which either criterion is imposed. These intermediate structures can then serve as benchmarks for comparison when a real image is analyzed.

This paper is organized as follows. Section 2 is comprised of a description of the GMST and GIST structures. It also contains brief summaries of the protocols used to form these trees, as well as methods for transitioning from the GIST towards the GMST. Section 3 contains basic statistical descriptions of the structures generated for a given set of graph realizations. Section 1 introduces the statistical measurements of edge density and tortuosity 
and outlines how they may be used to study an experimentally observed image. Finally, Section 5 has some concluding remarks.

\section{Models and algorithms}

Spanning trees were generated for graphs with 250,000 nodes arrayed on a square lattice connected by 500,000 edges. The nodes were divided into groups of 64 nodes each, with each group containing the nodes within an eight by eight square. The size of the groups was chosen such that the groups were large enough to allow many possible subtrees within a group, but still small enough to allow many groups within the graph. Each edge was randomly assigned a weight uniformly distributed between 0 and 1 . Twenty graph realizations were generated using a different random seed for each (i.e., a different set of edge weights). The choice of an underlying graph that conforms to the square lattice and groups that were spatially compact was made to allow the resulting trees to be visualized readily. The methods presented here, however, are general to any type of graph.

We have employed one of the heuristics developed by Dror et al. for generation of the GMST structures [1]. A brief summary of the algorithm that we have used is given below. The interested reader is referred to the original paper for a more detailed discussion of several possible protocols for producing GMST's. We have chosen to use heuristic H1 given by Dror et al. For any given graph (generally containing many loops), this method begins with a small tree (initially comprised of a single edge) connecting two groups via the most efficient path possible. The tree is then "grown" by adding the shortest path that enables the tree to span a new group (i.e., one not already spanned by the tree). This step is repeated until the tree spans all of the groups in the system.

\section{GMST algorithm:}

1. Initialize the tree by choosing a group randomly and connecting it to the closest neighboring group. Here closest means the group containing the node which can be added to the existing tree by the lowest weight path.

2. Find the group closest to the tree not already included in the tree and connect it to the tree.

3. If all of the groups are now spanned, terminate the algorithm, otherwise return to step 2 .

This algorithm was chosen because the specific details of our application allow it to be implemented efficiently. For example, because the graph we use has a straightforward geometric interpretation, it is simple to determine which group may be closest to the existing 
tree and what nodes within the tree are likely to be close to these groups. Thus, rather than identifying the minimum path to each region at each step, the distance to the majority of regions need not be calculated and further, for the regions that are checked, the distance from only a limited number of the nodes included within the tree must be calculated. Finally, as is discussed below, the size and regularity of the graph make it unnecessary to generate a distinct tree starting from each group and then find the tree with minimum total weight among these. Instead, a very small sample of groups is used as possible starting points and the minimum of these is designated the GMST.

As a basis of comparison for the globally minimized structure that the GMST protocol generates, we have defined a class of structures which we term generalized invasive spanning trees, or GIST's. GIST structures meet the same spanning conditions as GMST structures, but include local minimization in place of the GMST requirement of minimum total weight. The GIST structures are "grown" one edge at a time, analogously to the process of invasion percolation.

\section{GIST protocol:}

1. Begin with a tree comprised of the lowest weight edge in the underlying graph.

2. Add to the tree the lowest weight edge remaining that adjoins the existing tree.

3. If the newly added edge forms a loop with the edges already in the tree, delete it from the tree and from further possible additions.

4. If the tree now spans at least one node from each group, it is the GIST. Otherwise, return to step 2 .

The third step in the GIST protocol is included to ensure that a loop-less, tree structure is obtained, which is analogous to the creation of "trapped" regions in the trapping invasion percolation algorithm. With this exception, the protocol outlined above is identical to a standard invasion percolation protocol [17]. The only apparent difference is that standard invasion percolation algorithms terminate once the system is percolated (i.e., connected) in a few coordinate directions, rather than when it meets the spanning criterion given here. However, a change of language makes it clear that the spanning criterion is equivalent to a percolation condition of a rather unusual structure in high dimensional space. Consider a graph consisting of $2 K$ groups. Each group of nodes can be mapped to one face of a hypercube in $K$ dimensions. A percolating cluster in this arrangement, which includes no internal nodes, is one that connects all of the faces of the hypercube or, in other words, one which connects at least one node from each group. Thus the group spanning requirement for a graph whose nodes are divided into $2 K$ groups is equivalent to percolation of a hypercube 
whose nodes are confined to the faces of the cube in $K$ dimensional space. Note that this equivalence means that any of the highly-refined algorithms developed for the generation of invasion percolation networks (e.g. Sheppard et al. [20]) could be modified for use here affording tremendous computational speed-ups relative to the simple algorithm currently employed.

Of importance for the purpose of comparison with GMST structures is that the criterion for adding an edge to the growing GIST structure is based only upon a local condition. At every node in the system, the lowest weight edges will be included in the tree first, independent of which edge is most useful in meeting the "terminating" condition that requires all groups to be spanned. Thus the GIST represents the locally optimal extreme of all generalized spanning trees, while the GMST represents the globally optimal extreme. A careful comparison of the GIST and GMST protocols, however, reveals that under certain conditions the algorithms are identical. Specifically, the GMST protocol reduces to the GIST one for the situation in which each group is of cardinality 1. In other words, if each group is comprised of a single node, the closest new group is always connected to the existing tree by the lowest weight edge adjoining the tree, excluding those edges that would form a loop. For such graphs the GMST and GIST reduce to the MST and the acyclic invasion percolation network, respectively. The MST and acyclic invasion percolation structures have previously been shown to be identical [19, 18]. As the groups increase in size, however, the GIST and GMST structures diverge from one another.

As reported below, the structures generated by the GIST protocol are extremely dense by comparison to GMST structures and consequently difficult to compare directly. To address this issue we have adopted a simple method termed "pruning," which reduces the density of the GIST. In the pruning process, the edges comprising the GIST are sorted in order of non-increasing weight. Each "redundant" edge is then removed in turn, beginning with the highest weight edges. Here redundant is used in the same sense as in Dror et al., indicating an edge whose deletion leaves a spanning tree [1]. Indeed, this procedure is very similar to Heuristic H2 proposed by Dror et al. for the generation of a GMST from the MST of the same graph. Because (unlike the heuristic employed by Dror et al.) the initial tree in our use of the pruning algorithm is not an MST, the resulting tree is still quite far from the GMST. The result of repeatedly applying this algorithm to any initial tree is to reduce the density of the tree leading to a backbone structure. This is similar to the concept of identifying the (elastic or flow-carrying) backbone of a percolation cluster.

Finally, a second transforming protocol was employed to study structures whose properties are intermediate between the GMST and the backbone of the GIST. These intermediate structures serve as useful comparison points for branched networks that are a mixture of GMST and GIST backbone structures. Furthermore, investigating structures between the GIST backbone and the GMST allows us to assess the value of different statistics over a 
range of structures, rather than only at the extremal structures. This method is an adaptation of the third heuristic proposed by Dror et al. The procedure begins with any feasible spanning tree, which is then gradually converted to a more globally optimal structure.

\section{Conversion protocol:}

1. Begin with the backbone of the GIST.

2. Choose an arc (an unbranched chain of edges) within the tree.

3. Delete this arc and reconnect its end points with the path of lowest weight.

4. Return to step 2, until a termination criterion is met.

There are a few important observation that must be made regarding this protocol. The most important is that it is not able to transform the tree structure to the same extent as the heuristic employed by Dror et al. [1]. The main reason for this is the reconnection step (step 3) in this protocol fixes the end points of the new arc, whereas Dror et al. allow the replacement arcs to begin and end at arbitrary nodes within the tree. This is a compromise between the time complexity of the code and the efficiency of the resulting algorithm. Without this simplification, the time scaling of the code is too slow for use on the graph sizes considered here. In addition, the termination condition is also chosen to reduce computation time. The exhaustive search proposed by Dror et al. is more effective overall, but requires too much computational time to apply to the graphs used here. Instead, we have used a condition in which arcs are chosen randomly and replaced until 5000 successive replacements do not reduce the total weight of the spanning tree.

GMST structures took approximately 3 hours to generate on a single node of an IBM SP2 computer. GIST structures required roughly 4 minutes each to generate. Because of its comparative speed, the GIST routine was not optimized for execution time and could likely be accelerated considerably. The run time of the pruning algorithm depends directly on the size of the tree in the input. Runs on the initial GIST structures took approximately 6 minutes each, while runs on structures close to the GIST backbone took less than one minute. Finally, the conversion algorithm required approximately 10 hours per total conversion.

\section{Results and Standard Characterizations}

For each graph realization, GMST and GIST structures were generated. The GIST was then pruned repeatedly yielding a backbone structure, which was in turn reduced to a more globally minimal structure using the conversion protocol. As noted previously, the GMST protocol requires choosing a starting group. While the best possible GMST (within the limits 
of the heuristic method employed) requires testing every group as a potential starting point, in practice for our graphs this proved unnecessary. To test the variation in total weight caused by the choice of starting points, the GMST protocol was run for a single graph 100 times with a different starting point each time. In all cases, the final structures were closely related to one another (differing in less than $10 \%$ of included edges). Furthermore, the total weights of the trees were narrowly distributed, with a relative standard deviation of $0.2 \%$. As such, almost any starting group will yield a good approximation of the GMST. For the data presented here, we generated three potential GMST structures and selected the minimum of those as the GMST. Examples of the GIST and GMST are shown in Figure 2. The trees depicted are small samples of the entire tree structure. Also shown in the figure are the trees resulting from the pruning and conversion algorithms, which are labeled backbone and converted, respectively. Note that the GIST has a much higher density than any of the other structures, making direct comparisons with it difficult.

Figure 3 shows the total weights of several spanning trees for a single graph. Two features from this figure merit special mention. One feature is the gap in total weight between the result of the conversion protocol and the GMST. This difference highlights the limitations of the conversion protocol as formulated here. In principle, a more complete conversion could produce trees closer to the GMST, for example by following the protocol outlined by Dror et al. more faithfully. However, as noted above, the computational cost of such an approach was prohibitive. While the protocol given above for the conversion of the backbone structure calls for termination after a fixed number of consecutive arc replacements leave the structure unchanged, the conversion process may also be stopped after a fixed number of attempted replacements or after a set total weight has been passed. Either of these choices will result in a structure that is only partially converted. In other words, this will result in a structure that has some globally dictated characteristics, but still has significant degrees of local minimization incorporated.

The second notable feature is the large drop-off in total weight between the GIST and its backbone. This drop indicates that a significant majority of the edges in the GIST structure (over $80 \%$ ) play no role in meeting the spanning requirement. That many edges in the GIST can be removed is to be expected, in that edges are added to the GIST with no regard for the utility in creating a spanning tree. However, the degree to which edges can be removed is surprising. The pruning algorithm considers only the weight of an edge and its role in maintaining a single, connected spanning tree. This is a primarily local calculation and so it was originally expected that the backbone structure would be closely related to the GIST. Instead, the majority of edges in the GIST are pruned away in creating the backbone.

While the weights shown in Figure 3 are for a single graph, the variation in the weight of each tree between graphs is very small. This is not due to similarities in the actual trees, which display very few common edges. For example, less that $4 \%$ of the edges in the GMST 
for one graph are present in the GMST for any other graph. Instead, it suggests that the total weight of a GMST is insensitive to the specific details of the underlying graph. The total weights of each type of tree averaged over twenty different graph realizations are given in Table 1. Also listed in Table 1 are the average edge densities for each type of graph. The edge density is calculated as the number of edges included in the spanning tree divided by the number of edges in the complete graph. As expected, the edge density is strongly correlated with the total weight of each tree. Because the minimization of the total tree weight is the objective of the GMST protocol, the total tree weight can serve as an estimator of the degree to which a tree resembles the GMST.

Figure 4 shows the inclusion fraction distribution, or more briefly the inclusion fraction, which is defined as the fraction of edges in the underlying graph of a given weight included in each tree as a function of edge weight. For the GIST, the inclusion fraction has a sigmoidal dependence on edge weight. Recalling the previous discussion of the equivalence between spanning and percolation, it is useful to compare the inclusion fraction of the GIST to that of invasion percolation networks. The inclusion fraction for a sufficiently large (non-trapping) invasion percolation network is expected to take the form of a step function, with finite-size effects evidenced by deviations from the ideal function before and after the step [17, 21]. The GIST distribution displays near perfect step behavior at high edge weights, (with a vanishingly small number of high weight edges included) but a much more gradual drop-off at lower edge weights relative to an ideal step function. This behavior is reminiscent of an invasion percolation network with trapping [17, 21]. At large sizes, the inclusion fraction for this type of percolation network drops sharply beyond a critical value of edge weight (like a step function). At low edge weights, however, the inclusion fraction drops off gradually as the critical value is approached. The area between the inclusion fraction for the percolation network and the step function approaches a non-zero constant for large systems. This same type of low weight edge behavior is observed in the GIST inclusion fraction. A finite fraction of low weight edges are not included in the GIST because adding them would create a loop. In addition, there is a small area at the periphery of the graph (comprised of the most extreme groups) that is rarely visited by the tree. This finite-size effect is the reason the inclusion fraction of the GIST does not reach its limiting value of one at the lowest edge weights.

The inclusion faction for the backbone structure matches that of the GIST exactly at high edge weights. The few high weight edges included represent "bottlenecks" in the GIST, which are essential to the backbone. These bottlenecks should not be confused with the most vital edge of a spanning tree, which is the edge whose exclusion (and the subsequent reconnection of its end-points) would cause the greatest increase in total tree weight for a minimized tree [22], though they are likely to coincide to some degree. Instead, they occur when, in the course of creating the GIST, the invading tree reaches a point where all of the 
edges on the boundary of the current tree are high weight edges. One of these high weight edges must be included in the GIST and cannot be removed in the pruning process. Based on our simulations, this situation arises several times in the typical construction of a GIST. All of these instances are during the early stages of the tree formation, however, and so a vanishingly small fraction of the high weight edges is included in the limit of an infinitely large graph. It is interesting to note that the inclusion fraction for the backbone structure has an extended plateau at low edge weights. This suggests that low weight edges are equally likely to be included in the backbone structure nearly independent of their weight (below a threshold). This can be contrasted with the curve for the GMST, in which lower weight edges are included more frequently than higher weight edges for all edge weights. The inclusion fraction for the converted structure is intermediate between the backbone and the GMST. Although the distributions shown in Figure 4 differ significantly from one another, the mean weight of the edges included in each tree are very consistent (varying by a maximum of 0.02).

\section{Analysis and Discussion}

While the differences between the various types of trees in standard characterizations such as total weight or included edge fraction are clear, they are also of little utility in analyzing a single given structure. These measurements rely on the complete knowledge of the graph, including all edge weights. When considering a physical problem such as the infiltration of tumor cells into a porous gel, this level of information is generally difficult, if not impossible, to obtain. Instead a measurement relying only on the specific details of the observed structure and the most rudimentary information about the underlying graph is desirable. Furthermore, in such examples the complete structure of the tree is frequently difficult to image. Accordingly, a statistical measurement relying only on local information would be of particular value.

One measurement that meets these criteria is the occupied edge density, which measures the fraction of edges in the underlying graph that are included in the spanning tree, a measurement that only requires an estimate of the total number of edges in the underlying graph (along with the network structure). As shown in Figure 4 , the mean weight of the edges included in each tree varies little between different types of structures. Thus, the total weight of a tree correlates strongly with the fraction of edges in the underlying graph occupied by the tree. Another useful measurement is the tortuosity, $\tau$, of the arcs in the tree. Here tortuosity takes its common geometric meaning and is defined as an average of the ratio of the path length between two arbitrary nodes in the tree and the Euclidean distance between them. For a relatively large tree, this measurement can be made by averaging over the nodes included in the tree (or even a portion of the tree, if the tree is large enough). For 
small trees, however, an ensemble of trees would be necessary for an accurate measurement. This measurement can be made with no information about the underlying graph at all. For the trees considered here, the tortuosity is an increasing function of the path length.

$$
\tau(\ell)=\left\langle\frac{\text { Path length between two arbitrary nodes }}{\text { Euclidean distance between nodes }}\right\rangle
$$

In this equation, we have explicitly indicated that tortuosity should be measured for a single path length $\ell$, hereafter this notation will be suppressed and tortuosity will be indicated simply as $\tau$. The angular brackets indicate an average over pairs of nodes (and over structures, if more than one is given).

Figure 5(a) depicts average tortuosity curves for several types of spanning trees. The variation in the tortuosity curves between graph realizations is extremely small (the relative standard deviation is less than $0.1 \%$ ) and as such is not indicated on the plot. Note that the tortuosity curves are ordered such that the GIST tree is the most tortuous, while the GMST is the least tortuous, with the backbone structure and the converted structure falling in between. Figure 5(b) shows the relation between total tree weight and tortuosity. In this figure, the average total weight of each type of tree is plotted against the average tortuosity at a fixed path length. The length chosen here was twenty edge lengths, but similar plots can be made for any path length up to the lengths considered. Tortuosity and total weight are positively correlated for all of the trees considered. The tortuosity and total weight of the spanning trees vary almost linearly between the backbone and GMST structures. The GIST structure, however, has a very high total weight relative to the backbone structure that is not accompanied by a correspondingly large increase in tortuosity. This suggests that in some respects the structure of the backbone resembles that of the GIST more closely than is indicated by the difference in total weight. Here it is simply noted that this resemblance comports with our initial expectation that the pruning algorithm would not affect the structure of the GIST to a very significant degree. Further investigation is necessary to understand the relation between a GIST and the resulting backbone tree more completely.

Assessing the degree to which a tree has been formed under a global criterion requires more than just measuring the tortuosity (or occupied edge density). In particular, the measurements presented above are all specific to one type of graph - a square lattice with random and uniformly distributed edge weights. Thus the scale set by the GIST and GMST structures in the this work can only be used to evaluate trees that develop on underlying graphs with similar average coordination and distributions of edge weights. If a tree develops on a graph that has a different distribution of edge weights or different coordination number, new standards for the tortuosity (for example) of the GIST and GMST structures will need to be set. A simple example will serve to illustrate this point. Table 2 compares the weight 
and tortuosity of trees generated on several underlying graphs. The first data set is for the graph used in generating Figure 3. The second is for an identical graph except the edge weights are rescaled such that they span 0.45 to 0.55 , while maintaining the same ordering. Because the GIST considers only relative weights (i.e., which edge has the lowest weight) this rescaling does not affect the structure of the GIST. The GMST, however, is profoundly affected producing a tree that is significantly less tortuous at a fixed path length, though the total weight increases appreciably. Thus, a tree that developed on the second graph can have a very different balance of global and local influence than one developed on the first graph even though they both have the same tortuosity. For example, while a tortuosity of 1.32 would indicate a structure very close to the GMST of the first underlying graph, the same structure would have developed on the second underlying graph with a significant degree of local influence.

The need for standards (i.e. tortuosity measurements for the GIST and GMST) for the specific type of underlying graph presents a less serious obstacle than appears at first glance. In particular, it is not necessary to reproduce an exact replica of the real graph. As discussed above, the variation of tortuosity between different realizations of the same type of graph is extremely small. Thus, the only knowledge required to measure and evaluate the tortuosity of a tree is a statistical understanding of the underlying graph. In addition, computational experiments indicate that the exact form of the edge weight distribution also does not impact the tortuosity of the GMST significantly. To make this assessment, we have generated a GMST structure on a graph with edge weights drawn from a Gaussian distribution with mean 0.5. The Gaussian distribution was scaled such that the standard deviation matched that of a uniform distribution between 0 and 1 , with values below 0 or above 1 set to the appropriate extreme. The statistical properties of the GIST and GMST on this graph are listed in Table 2 as Graph 3. The results are very similar to those for a graph with uniformly distributed edge weights. While it is necessary to test several other types of edge weight distributions before the claim can be confirmed, these results indicate that tortuosity is not sensitive to the exact form of the edge weight distribution. Recalling that the structure of the GIST is not determined by the distribution of edge weights, this means that setting the tortuosity standards for a general class of underlying graphs can be accomplished with very limited information about the specific graph.

In practical terms, given a single tree structure (or an ensemble of small structures), a simple procedure can be followed to estimate its relation to the GMST and GIST.

\section{Evaluation protocol}

1. Measure the tortuosity, $\tau$, for the given tree at a fixed path length.

2. Generate the GIST and GMST for the underlying graph thought to exist in the problem being considered and measure their tortuosities. 
(a) Estimate the coordination number of the expected underlying graph. Generate a model underlying graph, with the same type of coordination. For example, expecting a coordination number of 6 , one could use a triangular lattice. Estimate the length scale of any long-range effects in the system and group the nodes in the underlying graph in accordance with this length scale.

(b) Using any distribution of edge weights, generate the GIST and measure its tortuosity, $\tau_{G I S T}$.

(c) Estimate the relative dispersion of the edge weights in the graph. This requires an approximation of the ratio $\sigma / \mu$, where $\sigma$ is the standard deviation and $\mu$ is the mean of the edge weights.

(d) For the underlying graph chosen in step 2(a), generate a graph realization whose edge weights are drawn from a distribution with the relative dispersion estimated in step 2(c). Generate the GMST for this graph realization and measure its tortuosity, $\tau_{G M S T}$.

3. Evaluate the ratio

$$
\bar{\tau}=\frac{\tau-\tau_{G M S T}}{\tau_{G I S T}-\tau_{G M S T}}
$$

which represents the degree to which the given tree resembles the GIST. For example, a value of 0.1 would indicate a structure that is primarily globally optimal (GMST-like), while 0.8 would indicate a structure that is dominated by local effects (GIST-like).

As an example, consider the problem of cells moving through a porous medium under the influence of a nutrient gradient. The pores in the medium play the role of edges in the graph and their intersections are the nodes. Assume that we can measure the coordination number of the pore structure, perhaps by the same imaging technique that has produced the tree we are analyzing. For a medium with a coordination number of three, we might choose a hexagonal lattice as our graph. The long-range effect we are investigating is the influence of the nutrient gradient, so groups in the graph should be the size of the nutrient diffusion length scale. So our underlying graph would now be a hexagonal lattice, tiled into groups each of which has the same length scale as the nutrient diffusion length in the real system. Using this graph, we can use any edge weight distribution we choose and generate the GIST. To generate the GMST, however, it is necessary to make one more estimate - we must decide what type of resistances are present. For this problem, the resistance might be caused by the cells squeezing to fit into the pores. Thus the weight of an edge would be the inverse of the pore diameter. After estimating the range of pore diameters in the porous medium, we can construct an edge weight distribution that matches this estimate. Using this distribution, we can complete our graph realization and measure the tortuosity of the 
GMST. Note that the only information required in this process was very general and should be relatively easily accessible experimentally.

\section{Conclusions}

The results described above show that altering the criterion for including edges in a spanning tree from a global one (the GMST) to a local criterion (the GIST) has a measurable impact on the statistical characterization of the resulting trees. In particular, the total weight, occupied edge density, inclusion fraction distribution, and the tortuosity varied systematically with different types of spanning trees. Of these, however, the majority require extensive information about the graph under consideration, including individual edge weights. In contrast, however, measurement of the tortuosity of a tree only requires information about the structure of the tree. Measurement of the occupied edge density requires minimal information about the underlying graph, in addition to the tree structure, but does not require the detailed information necessary for measurements like the total weight or the inclusion fraction distribution. As noted above, both the edge density and the tortuosity measurements for a single network must be made in the context of the type of underlying graph present. We emphasize that while this context requires some basic knowledge of the characteristics of the underlying graph, this is a much more accessible level of information than the complete information required for measurements such as the total weight of the tree.

Using tortuosity to characterize a network has one additional advantage. The tortuosity is measured along individual arcs for relatively small path lengths. Thus the tortuosity of a structure can be measured accurately even if only a small portion of the structure is observed. This is of particular value in assessing systems that are challenging to image completely. These features make the tortuosity and occupied edge density promising tools for the investigation of naturally occurring tree structures whose temporal formation cannot be observed directly.

One type of variation that is not addressed in the discussion above is a change in the coordination number of the graph. It has been shown that changing the coordination of the underlying graph has a pronounced impact on the percolation threshold in the invasion percolation system [17]. As such, it is expected that similarly pronounced impacts would be observed on the structure of any spanning tree. Characterizing the effect of different coordination numbers on the structure of the GMST, however, follows the exact methodology

outlined in this paper. In sum, employing the protocols discussed previously on a graph of interest will yield a set of benchmark tortuosity or occupied edge density measurements that may then be used to assess whether global or local weight criteria played an important role in the development of any given tree structure. 


\section{Acknowledgements}

This work has been supported in part by grants CA84509 and CA69246 from the National Institutes of Health. The work was also supported by the Engineering Research Program of the Office of Basic Energy Sciences at the Department of Energy (Grant DE- FG0292ER14275). The authors would like to thank Dr. T. S. Deisboeck for valuable discussions.

\section{References}

[1] M. Dror, M. Haouari, and J. Chaouachi. Generalized spanning trees. Eur. J. Oper. Res., 120:583-592, 2000.

[2] I. Rodriguez-Iturbe and A. Rinaldo. Fractal River Basins. Cambridge University Press, Cambridge, 1997.

[3] A. Rinaldo, I. Rodriguez-Iturbe, R. Rigon, E. Ijjasz-Vasquez, and R. L. Bras. Selforganized fractal river networks. Phys. Rev. Lett., 70:822-825, 1993.

[4] T. Sun, P. Meakin, and T. Jossang. Minimum energy dissipation model for river basin geometry. Phys. Rev. E, 49:4865-4872, 1994.

[5] A. Maritan, A. Rinaldo, R. Rigon, A. Giancometti, and I. Rodriguez-Iturbe. Scaling laws for river networks. Phys. Rev. E, 53:1510-1515, 1996.

[6] D. R. Kayser, L. K. Aberle, R. D. Pochy, and L. Lam. Active walker models: Tracks and landscapes. Physica A, 191:17-24, 1992.

[7] R. D. Freimuth and L. Lam. Active walker models for filamentary growth patterns. In L. Lam and V. Naroditsky, editors, Modeling Complex Phenomena, pages 302-313. Springer, New York, 1992.

[8] G. B. West, J. H. Brown, and B. J. Enquist. A general model for the origin of allometric scaling laws in biology. Science, 276:122-126, 1997.

[9] T. S. Deisboeck, M. E. Berens, A. R. Kansal, S. Torquato, A. O. Stemmer-Rachamimov, and E. A. Chiocca. Patterns of self-organization in tumour systems: Complex growth dynamics in a novel brain tumour spheroid model. Cell Prolif., 34:115-134, 2001.

[10] M. Schwartz. Computer and Communications Network Design and Analysis. PrenticeHall, Englewood Cliffs, NJ, 1977. 
[11] B. Gavish. Formulations and algorithms for the capacitated minimal directed tree problem. J. ACM., 30:118-132, 1983.

[12] C. Adami and A. Mazure. The use of minimal spanning tree to characterize the 2D cluster galaxy distribution. Astron. Astrophys. Sup., 134:393-400, 1999.

[13] J. B. Kruskal Jr. On the shortest spanning subtree of a graph and the traveling salesman problem. Proc. Amer. Math. Soc., 7:48-50, 1956.

[14] R. C. Prim. Shortest connection networks and some generalizations. Bell Sys. Tech. J., 36:1389-1401, 1957.

[15] S. C. Narula and C. A. Ho. Degree-constrained minimum spanning tree. Comput. Op. Res., 7:239-249, 1980.

[16] G. Zhou and M. Gen. A note on genetic algorithms for degree-constrained spanning tree problems. Networks, 30:91-95, 1997.

[17] D. Wilkinson and J. F. Willemsen. Invasion percolation: A new form of percolation theory. J. Phys. A: Math. Gen., 16:3365-3376, 1983.

[18] A. L. Barabasi. Invasion percolation and global optimization. Phys. Rev. Lett., 76:37503753, 1996.

[19] K. S. Alexander. Percolation and minimal spanning forests in infinite graphs. Ann. Probab., 23:87-104, 1995.

[20] A. P. Sheppard, M. A. Knackstedt, W. V. Pinczewski, and M. Sahimi. Invasion percolation: New algorithms and universality classes. J. Phys. A.: Math. Gen., 32:L521-L529, 1999.

[21] D. Wilkinson and M. Barsony. Monte-Carlo study of invasion percolation clusters in 2 dimensions and 3 dimensions. J. Phys. A.: Math. Gen., 17:L129-L135, 1984.

[22] L. H. Hsu, R. H. Jan, Y. C. Lee, C. N. Hung, and M. S. Chern. Finding the most vital edge with respect to minimum spanning tree in weighted graphs. Inf. Process. Lett., 39:277-281, 1991. 


\section{Figure Captions}

Figure 1: Example of a weighted graph and the resulting minimal spanning tree. (a) shows all of the edges and nodes in a graph, with the weight of each edge indicated next to the edge. Graph edges are depicted by broken lines. (b) shows the minimal spanning tree for this graph, which is the set of edges that connects every node in the graph in the tree with the lowest total weight. Edges included in the tree are shown as solid lines, while edges not included remain broken lines. The total weight of the tree in (b) is 40, and the occupied edge density (number of edges included in the tree divided by total number of edges in the graph) is $15 / 25=0.6$. (c) shows the invasion percolation network for the same graph. Note that the invasion percolation network may have loops and in this case there are two closed loops. If loop formation is prevented (resulting in the highest weight edge in any loop remaining unoccupied) the result is the acyclic invasion percolation network. As can be readily seen by comparing figures (b) and (c) the acyclic invasion percolation network is identical to the MST.

Figure 2: Examples of the (a) GIST, (b) GMST, (c) backbone, and (d) converted structures for a single graph. The backbone structure is the result of repeatedly applying the pruning algorithm to the GIST. The converted structure is the result of applying the conversion algorithm to the backbone structure. In each case a small, representative section is shown (not the entire tree). The underlying graph is not shown in these images.

Figure 3: The total weight of several types of trees for a single graph realization. Inset is a magnification of the three low weight trees to highlight the relative difference. The GIST, backbone, and converted structures fall along a continuum of structures as indicated by the connecting line. The backbone structure is generated by repeatedly pruning the GIST. The converted structure is the result of applying the conversion protocol to the backbone structure. Spanning trees between the converted tree and the GMST have yet to be produced.

Figure 4: Plot of the fraction of edges included in each tree as a function of edge weight. The mean weight of the edges included in each tree is indicated by the circle on each distribution.

Figure 5: (a) Tortuosity versus path length for several types of trees averaged over twenty graph realizations. The variation between graphs is sufficiently small that the curve for each graph is indistinguishable from the averaged curve. Note that the ordering of the curves corresponds to the total tree weights (i.e., high tortuosity correlates with high total weight). This correlation is explicitly displayed in (b). Inset is a magnification of the low total weight points, emphasizing their relative differences. Also included in the inset are several intermediate structures that are generated during the conversion algorithm. 
Table 1: Total weight and edge density of different types of spanning trees averaged over twenty graphs. As indicated in Figure 3, the backbone structures result from pruning the GIST, and in turn are used to produce the converted trees.

\begin{tabular}{l|rl|ll}
\hline Structure & \multicolumn{2}{|c|}{ Weight } & \multicolumn{2}{c}{ Edge density } \\
\hline GIST & 54000 & \pm 1400 & 0.4539 & $\pm 2.1 \times 10^{-2}$ \\
\hline Backbone & 8650 & \pm 67 & 0.0766 & $\pm 4.9 \times 10^{-4}$ \\
\hline Converted & 7120 & \pm 82 & 0.0630 & $\pm 6.2 \times 10^{-4}$ \\
\hline GMST & 6350 & \pm 35 & 0.0541 & $\pm 2.3 \times 10^{-4}$ \\
\hline
\end{tabular}


Table 2: Comparison of the GIST and GMST on three different graphs. All three graphs are square lattice, with different edge weight distributions. The tortuosity is measured at a fixed path length of 20. The second graph is identical to the first, except its edge weights have been rescaled to lie between 0.45 and 0.55 (versus 0 and 1.0 in the first graph). The edge weights in the third graph have been converted to follow a Gaussian distribution with the same mean and standard deviation as the first graph. The rescalings do not affect the structure of the GIST at all, though the total weight can change. In contrast, the GMST structure is dependent of the edge weight distribution used. However, comparison of the first and third graphs reveals that there may not be a strong dependence on the exact form of the distribution.

\begin{tabular}{l|l|r|r|}
\hline \multicolumn{2}{|c|}{} & Weight & Tortuosity \\
\hline Graph 1 & GIST & 55080 & 1.9175 \\
\cline { 2 - 4 } & GMST & 6341 & 1.3055 \\
\hline Graph 2 & GIST & 107637 & 1.9175 \\
\cline { 2 - 4 } & GMST & 10239 & 1.1365 \\
\hline Graph 3 & GIST & 58361 & 1.9175 \\
\cline { 2 - 4 } & GMST & 6350 & 1.3101 \\
\hline
\end{tabular}




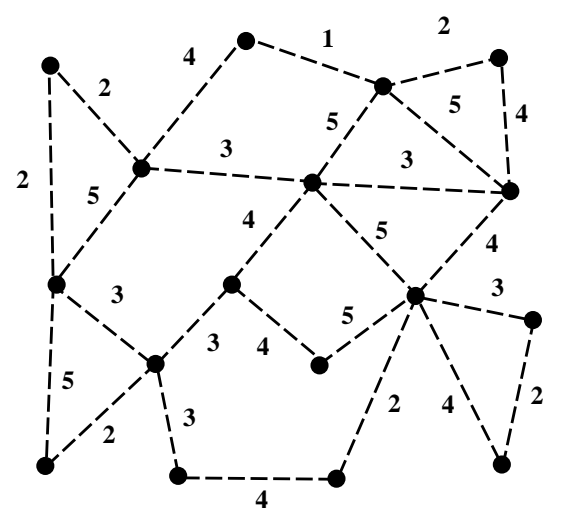

(a)

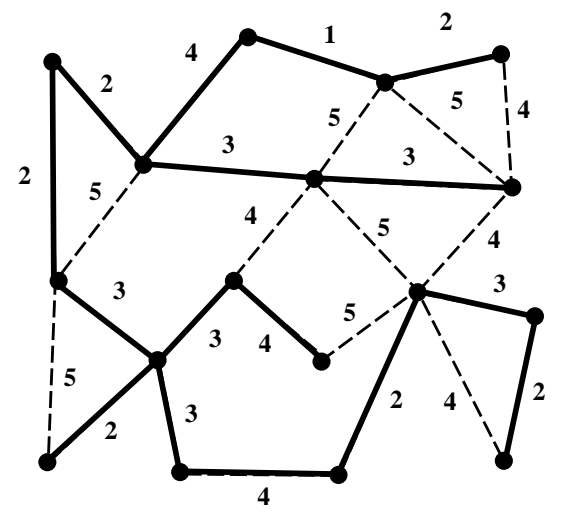

(b)

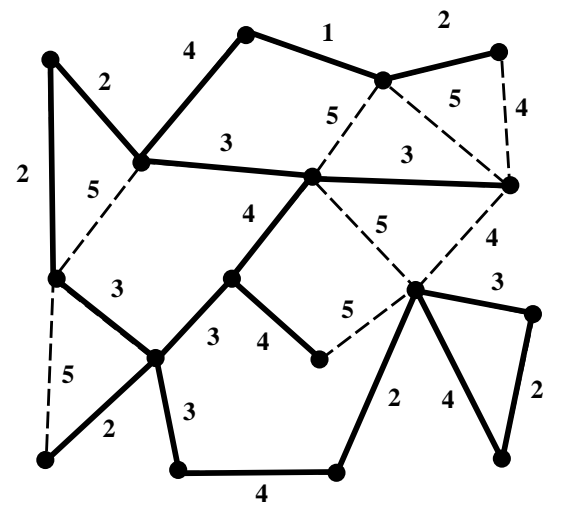

(c)

20

Figure 1: 


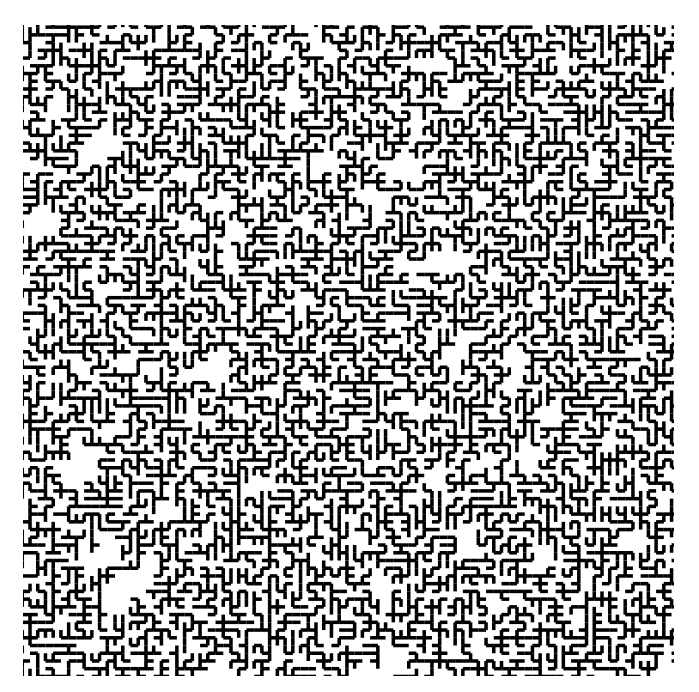

(a)

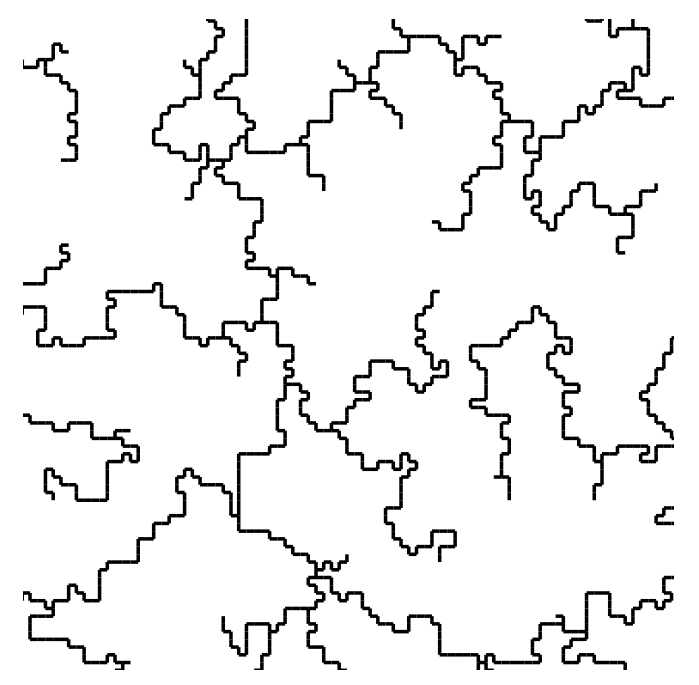

(c)

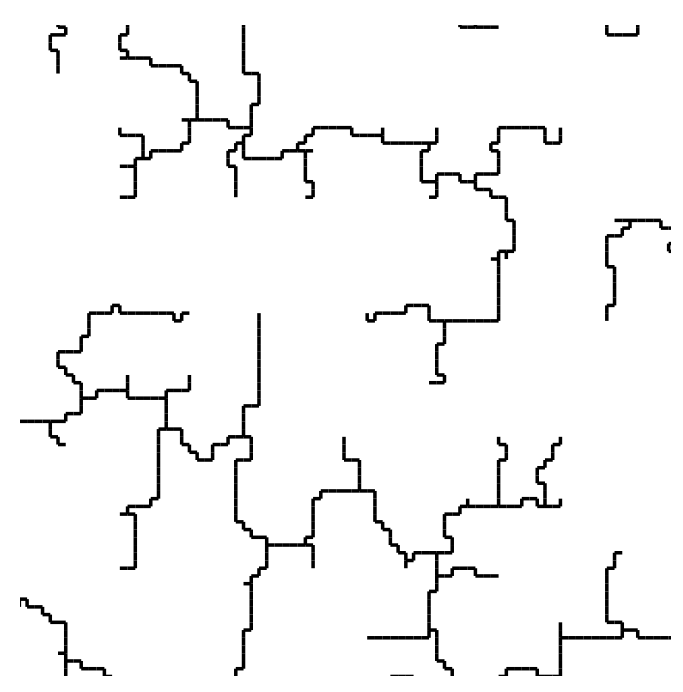

(b)

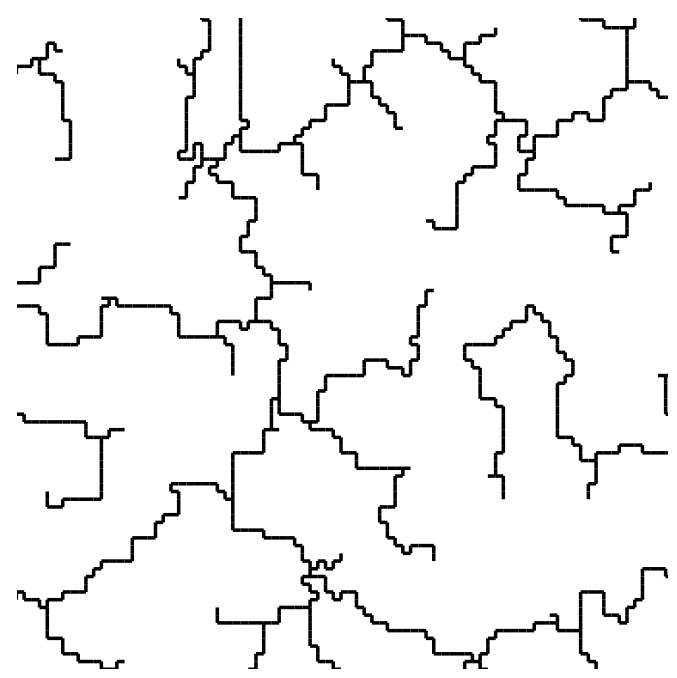

(d)

Figure 2: 


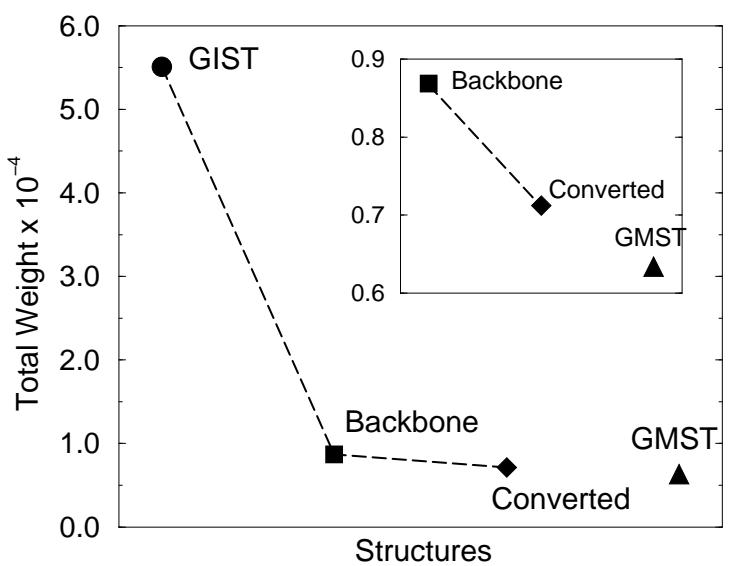

Figure 3:

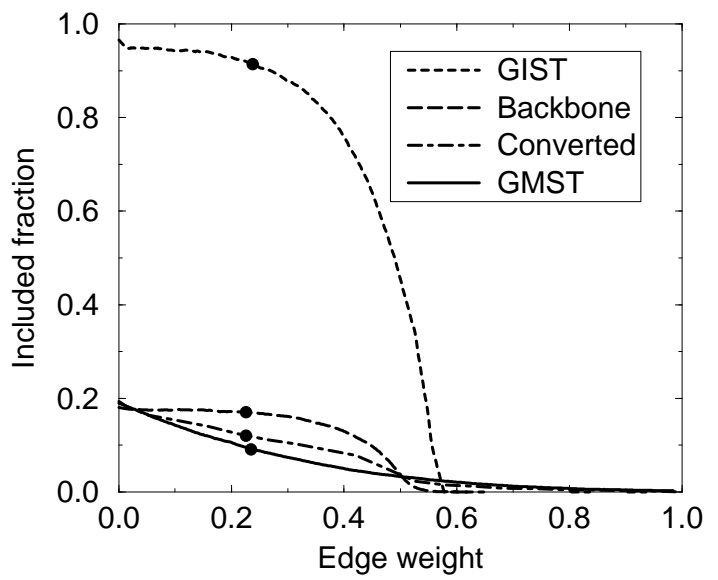

Figure 4: 


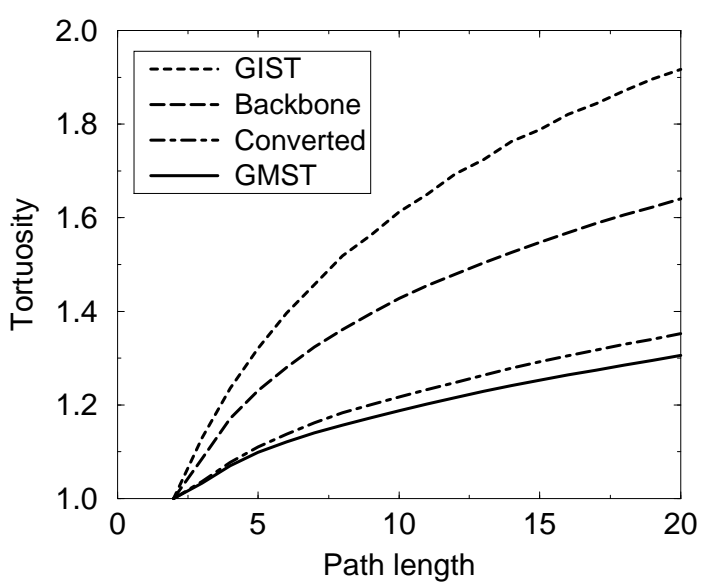

(a)

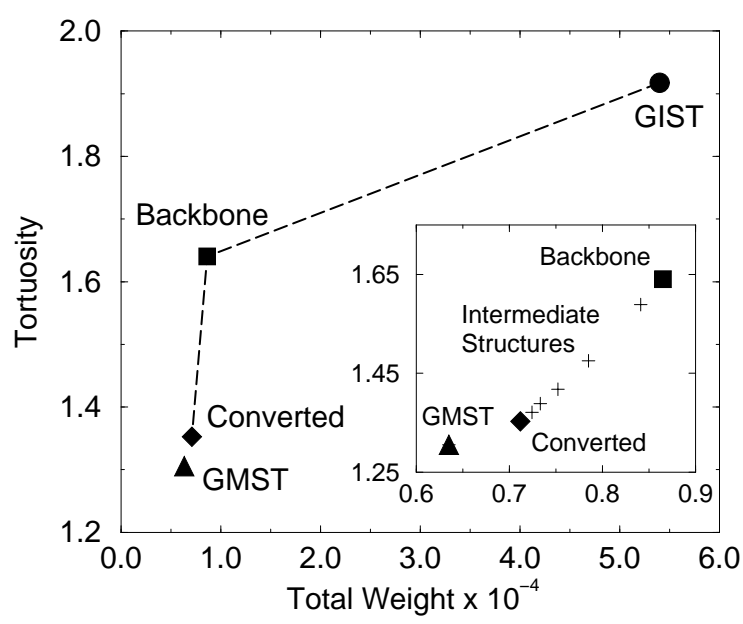

(b)

Figure 5: 